\title{
Does hyperpriming reveal impaired spreading of activation in schizophrenia?
}

Laurent Lecardeur ${ }^{\mathrm{ab}}$, Bénédicte Giffard ${ }^{\mathrm{b}}$, Mickael Laisney ${ }^{\mathrm{b}}$, Perrine Brazo ${ }^{\mathrm{ac}}$, Emmanuel Stip $^{\mathrm{d}}$, Francis Eustache ${ }^{\mathrm{b}}$, Sonia Dollfus ${ }^{\mathrm{ac}}$

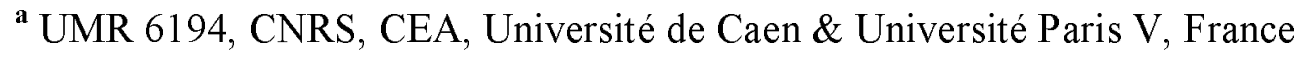

${ }^{\text {b }}$ Unité E0218 INSERM, EPHE, Université de Caen, France

' Department of Psychiatry, Centre Hospitalier Universitaire, Avenue Côte de Nacre, 14033 Caen, France

d Department of Psychiatry, Centre de Recherche Fernand Seguin, Hôpital Louis-H. Lafontaine, Université de Montréal, Montréal, Québec, Canada

\section{Letter to the editor}

Submitted to Schizophrenia Research in June 2007

\section{Revision submitted in August 2007}

\section{Corresponding author}

Laurent Lecardeur

Groupe d'Imagerie Neurofonctionnelle

GIP Cyceron Bd H. Becquerel, BP 5229

14074 Caen Cedex France

Phone: (33) 231470156

Fax: (33) 231470222

E-mail: lecardeur@cyceron.fr

Text: 995 words

One table 


\section{Dear Editors,}

Enhancement of semantic priming effects (hyperpriming) has been well documented in schizophrenic patients (Minzenberg et al. 2002). In a previous study, we demonstrated that hyperpriming was related to a more pronounced requirement to inhibit unrelated information (Lecardeur et al. 2007). On the other hand, several authors have suggested that hyperpriming could reflect enhanced spreading of semantic activation in schizophrenia (Spitzer et al. 1993; Spitzer 1997; Moritz et al. 2001). So far, there are few reports that attempted to study the spreading of activation in schizophrenic patients compared to healthy controls, when the links between semantically related information were manipulated. Notably, authors stated that activation could consequently spread farther and further in the semantic networks of schizophrenic patients. Moreover, enhanced spreading of activation was hypothesized to be responsible of the appearance of thought and language disorders in schizophrenic patients. Consequently, we examined whether the manipulation of the type of connection and the semantic distance between prime and target can modulate semantic priming effects in schizophrenic patients compared to healthy controls.

We recruited 30 schizophrenic outpatients meeting DSM-IV criteria for schizophrenia (American Psychiatric Association 1994) and 30 healthy controls, matched one by one for sex, age and education level. Verbal IQ did not differ between patients (Mean \pm standard deviation; 93.6 \pm 12.9$)$ and controls $(90.9 \pm 15.06)$. On the other hand, patients displayed significant motor slowing (355.6 \pm 73.1$)$ compared to controls $(296.1 \pm 39.5)$ as assessed by simple reaction time task $\left(t_{29}=4.85, p<0.001\right)$. Patients were stabilized, with low severity of symptoms (total score of PANSS: $46.96 \pm 3.23$ ) and all were treated with antipsychotics. All gave informed written consent, and the local ethical committee approved the study. 
To test the spreading of semantic activation between related primes and targets, semantic priming (SP) was assessed using two lexical decision tasks based on Lecardeur et al. (2007), one that favored only automatic process and the other that required both automatic and controlled processes. Experimental material contained four types of prime/target pairs: related, unrelated, those having the word "neutral" as prime, and those with a pseudo-word as target. Related pairs were selected according to a restricted verbal association task: 233 healthy students gave either the first coordinate or the first attribute that came to their mind in response to an inductor word. As a result, half of targets were coordinates of the prime (dress - skirt) and half were attributes of the prime (boat - oar). Targets were classified into two subcategories, according to their semantic distance from the prime: associations of first and second orders (words whose frequency of occurrence was the highest) were classified as semantically close pairs, while associations of fourth and fifth orders (the lowest frequency of occurrence) were classified as remote ones (Richards et Chiarello 1995). In this way, we obtained close coordinates (alligator - crocodile), remote coordinates (turnip - beet), close attributes (cape - eyeshade) and remote attributes (bear-fur). SP effects were obtained for each subject, subtracting response times for related targets from response times for unrelated targets (response times unrelated - response times related).

SP effects were analysed by four-way repeated measures analysis of covariance (ANCOVA), with group (patients, controls) as the between-subject factor, task (automatic, automatic/controlled), connection (coordination, attribution) and distance (close, remote) as within-subject factors (Table 1). Numerous studies stated that semantic priming of schizophrenic patients could be altered by their motor slowing (Minzenberg et al., 2002 for review). Consequently, we evaluated motor slowing in schizophrenic patients, thanks to a simple reaction time task. To take into account the motor slowing during priming tasks, we used the reaction times of patients and controls as covariates in repeated measures analysis of 
covariance. Group x connection $(F(1,448)=2.25, p=0.14)$ and group $\mathrm{x}$ distance $(F(1,448)=$ $0.009, p=0.92)$ interactions did not reach significance. A Group main effect was significant $(F(1,448)=7.44, p=0.008)$; The semantic priming effect was greater in patients $(217.91 \pm$ 37.91) than in controls $(110.69 \pm 60.9)$. A task main effect was significant $(F(1,448)=26.34$, $p<0.001)$; The semantic priming effect was greater in the automatic/controlled task $(255.05$ $\pm 290.23)$ than in the automatic task $(73.53 \pm 89.74)$. A connection main effect was also found $(F(1,448)=12.46, p<0.001)$. The semantic priming effect was greater for coordinates $(182.43 \pm 167.35)$ than for attributes $(146.01 \pm 165.46)$. However, the distance main effect did not reach significance $(F(1,448)=0.51, p=0.478)$.

The results demonstrated hyperpriming in patients compared to healthy controls. They suggested that the hyperpriming could not be state-dependent as stated previously (GouzoulisMayfrank et al. 2003), since the schizophrenic patients of the present study presented a low level of severity of symptoms. However, the manipulation of the type of connection and the semantic distance between related primes and targets did not allow us to differentiate patients and controls according to SP effects. The current findings suggested that semantic activation did not differently spread in semantic networks in schizophrenic patients compared to healthy controls. These results did not support the hypothesis of an enhancement of spreading of activation with related information in schizophrenia (Spitzer et al. 1993; Moritz et al. 2001), since the semantic distance did not influence the SP. Consequently, hyperpriming can be reported without enhanced spreading of activation in schizophrenic patients. Despite hyperpriming was equally reported in Alzheimer's disease (AD) patients, this effect does not rely on the same pathophysiology. In AD patients, enhanced priming effect is induced by a progressive loss of semantically related information (Giffard et al. 2001; Giffard et al. 2002): the distinctive attributes of concepts (stripe-tiger) are lost first with preservation of coordinate concepts (tiger-lion). Since distinctive attributes of concepts are lost, coordinate concepts 
become nearly identical and generate enhanced priming effects in AD patients compared to healthy subjects. On the contrary, hyperpriming in schizophrenic patients could not be produced from a degradation of semantically related information, since patients' and controls' SP did not differ on the type of connection. Consequently, the major result of this study suggests a preservation of the semantic verbal memory in schizophrenic patients.

\section{Acknowledgments}

This study was supported by a grant from the Centre Hospitalier et Universitaire of Caen and the Conseil Régional of Basse-Normandie (France). The authors would like to acknowledge P. Delamillieure, B. Mazoyer, N. Tzourio-Mazoyer, P. Maillard, and G. Perchey for comments on the manuscript and for technical assistance. 
American Psychiatric Association, 1994. DSM-IV: Diagnostic and Statistical Manual of Mental Disorders. 4th. The Association, Washington DC..

Giffard,B., Desgranges,B., Nore-Mary,F., Lalevee,C., Beaunieux,H., de la Sayette,V., Pasquier,F., and Eustache,F., 2002. The dynamic time course of semantic memory impairment in Alzheimer's disease: clues from hyperpriming and hypopriming effects. Brain. $125,2044-2057$.

Giffard,B., Desgranges,B., Nore-Mary,F., Lalevee,C., de la Sayette,V., Pasquier,F., and Eustache,F., 2001. The nature of semantic memory deficits in Alzheimer's disease: new insights from hyperpriming effects. Brain. 124, 1522-1532.

Gouzoulis-Mayfrank,E., Voss,T., Morth,D., Thelen,B., Spitzer,M., and Meincke,U., 2003. Semantic hyperpriming in thought-disordered patients with schizophrenia: state or trait?--a longitudinal investigation. Schizophr Res. 65, 65-73.

Lecardeur,L., Giffard,B., Laisney,M., Brazo,P., Delamillieure,P., Eustache,F., and Dollfus,S., 2007. Semantic hyperpriming in schizophrenic patients: Increased facilitation or impaired inhibition in semantic association processing? Schizophr Res. 89, 243-250.

Minzenberg,M.J., Ober,B.A., and Vinogradov,S., 2002. Semantic priming in schizophrenia: a review and synthesis. Journal of the International Neuropsychological Society. 8, 699-720.

Moritz,S., Mersmann,K., Kloss,M., Jacobsen,D., Wilke,U., Andresen,B., Naber,D., and Pawlik,K., 2001. 'Hyper-priming' in thought-disordered schizophrenic patients. Psychol Med. 31, 221-229.

Richards,L. and Chiarello,C., 1995. Depth of associated activation in the cerebral hemispheres: mediated versus direct priming. Neuropsychologia. 33, 171-179.

Spitzer,M., 1997. A cognitive neuroscience view of schizophrenic thought disorder. Schizophr Bull. 23, 29-50.

Spitzer,M., Braun,U., Maier,S., Hermle,L., and Maher,B.A., 1993. Indirect semantic priming in schizophrenic patients. Schizophr Res. 11, 71-80. 
Table 1. Semantic priming effects ${ }^{a}$ in milliseconds.

\begin{tabular}{|c|c|c|c|c|c|c|c|c|}
\hline & \multicolumn{4}{|c|}{ Schizophrenic patients $(n=30)$} & \multicolumn{4}{|c|}{ Healthy controls $(n=30)$} \\
\hline & \multicolumn{2}{|c|}{ Automatic task } & \multicolumn{2}{|c|}{ Automatic / controlled task } & \multicolumn{2}{|c|}{ Automatic task } & \multicolumn{2}{|c|}{ Automatic / controlled task } \\
\hline & Mean SP & (SD) & Mean SP & (SD) & Mean SP & (SD) & Mean SP & (SD) \\
\hline Remote attributes & 94.26 & $(190.7)$ & 286.1 & $(522.01)$ & 62.32 & $(67.97)$ & 138.67 & $(86.63)$ \\
\hline Close attributes & 46.91 & $(136.44)$ & 338.66 & $(448.83)$ & 40.18 & $(43.88)$ & 159.31 & $(97.71)$ \\
\hline Remote coor dinates & 115.08 & $(166.95)$ & 353.56 & $(387.58)$ & 67.62 & $(63.88)$ & 158.19 & $(94.37)$ \\
\hline Close coordinates & 82.48 & 237.63 & 422.29 & $(428.34)$ & 74.98 & $(47.4)$ & 183.93 & $(103.92)$ \\
\hline
\end{tabular}

${ }^{\text {a }}$ Semantic priming effect: [(mean RT unrelated) - (mean RT related)]

Semantic Priming (SP)

Standard Deviation (SD 
